\title{
Effectiveness of Superb Microvascular Imaging for the differentiation of intraductal breast lesions
}

\author{
Suleyman Bakdik, Serdar Arslan², Fatih Oncu², Mehmet Sedat Durmaz ${ }^{2}$, Aysegul Altunkeser², \\ Mehmet Ali Eryilmaz ${ }^{3}$, Yasar Unlu ${ }^{4}$
}

${ }^{1}$ Department of Radiology, Necmettin Erbakan University School of Medicine, ${ }^{2}$ Department of Radiology, University of Health Science, Konya Training and Research Hospital, ${ }^{3}$ Department of General Surgery, University of Health Science, Konya Training and Research Hospital, ${ }^{4}$ Department of Pathology, University of Health Science, Konya Training and Research Hospital, Konya, Turkey

\begin{abstract}
Aims: The aim of this study was to compare the diagnostic performance of superb microvascular imaging (SMI) and power Doppler imaging (PDI) for the differentiation of intraductal breast lesions. Materials and methods: A total of 54 intraductal breast lesions (39 benign, 10 atypical, and 5 malignant) in 53 patients were examined using B-mode ultrasonography, PDI, and SMI. Vascularity grading, distribution of microvessels, and penetrating vessels were evaluated using each Doppler technique. The diagnostic performances of both methods were compared. Results: SMI was more efficient in detecting flow signals than PDI $(\mathrm{p}=0.004)$. The highest diagnostic accuracy rates were achieved with SMI using vascular grading. When hypervascularity was used as a cut-off value to differentiate malignant and atypical lesions from benign lesions, the sensitivity, specificity, positive predictive value, negative predictive value, and accuracy were $66.6 \%, 80.7 \%, 66.6 \%, 80.7 \%$, and $75.6 \%$ for PDI, and $86.6 \%, 76.9 \%, 68.4 \%, 90.9 \%$, and $80.4 \%$ for SMI, respectively. Conclusions: SMI is more sensitive than PDI for detecting subtle blood flow in intraductal breast lesions with statistical significance. This novel and promising vascular imaging technique may be helpful in B-mode ultrasonography to distinguish intraductal breast lesions.
\end{abstract}

Keywords: superb microvascular imaging; power Doppler imaging; breast cancer; ultrasonography

\section{Introduction}

Intraductal breast lesions have a broad spectrum of pathologies and diverse imaging features. It is difficult to diagnose these lesions as benign, atypical, or malignant based on radiological findings [1]. B-mode ultrasonography (US) features cannot provide adequate diagnostic accuracy to predict the benignity or malignancy of the lesions [2] and for this reason, additional imaging methods are needed. If vascularity, which is important in the differentiation of breast lesions, could be evaluated in a

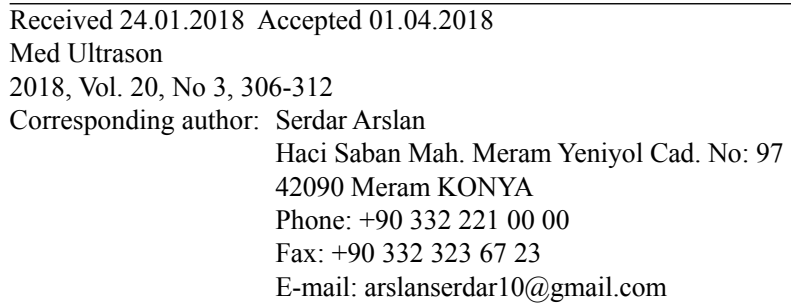

more detailed way, it would increase the diagnostic efficacy of B-mode US [3]. Malignant breast lesions demonstrate rapid growth, which requires nutrition supplied by newly formed microvessels in a process called angiogenesis [4]. Hence, malignant breast lesions have more neovascularization than benign breast lesions.

Power Doppler imaging (PDI) has often been used to detect blood flow in breast lesions using the integral backscatter signal of red blood cells. However, this method is insufficient for identifying subtle low-flow signals [5]. Superb microvascular imaging (SMI) is a recently developed Doppler technique; the underlying principle is a powerful and intelligent algorithm that efficiently separates low-speed flow signals from motion artifacts so that it can reveal low-flow signals in microvessels $[6,7]$.

The aim of this study was to compare the diagnostic performances of the SMI and PDI methods in differentiation of intraductal breast lesions. 


\section{Material and methods}

\section{Patients}

After the study was approved by the local ethics committee, informed oral and written consents were obtained from all patients. Fifty-four intraductal breast lesions detected in 53 female patients (mean age, 48.5 \pm 11.2 , range, 21-74 years) were included in this prospective study, which was conducted between February 2016 and July 2017. The criteria for inclusion in the study were the visibility of the lesion in B-mode US and no history of breast surgery or radiotherapy. The exclusion criteria were non-intraductal breast masses, severe organ failure, and an absence of histopathological results.

\section{Imaging analysis}

All patients were examined using B-mode US, PDI, and SMI using a Toshiba Aplio 500 (Toshiba Medical System Corporation, Tokyo, Japan) with a 7.2 to $14 \mathrm{MHz}$ high-frequency linear-array transducer. All sonographic scanning was performed separately by 2 independent radiologists, each with at least 8 years of experience in breast US.

During B-mode US evaluation, patients were placed in the supine position with the arms elevated. Transverse and sagittal views were acquired through routine scans, and grayscale features of the lesions were recorded. According to the B-mode US findings, the shape, margin, echo pattern, posterior acoustic features, ductal dilatation, and calcifications were recorded.

Subsequently, PDI and SMI were performed in order to evaluate the vascularity of the intraductal lesions. The probe was placed lightly on the lesion to avoid pressure on the microvessels. The region of interest was adjusted to cover the lesion and surrounding breast tissue. For PDI examinations, the scale was set at $5 \mathrm{~cm} / \mathrm{s}$, the mechanical index at 1.5 , the wall filter at $50-100 \mathrm{~Hz}$, and the frame rate at 10-25 frames/s. For SMI examinations, the scale was set at $1.5-2.5 \mathrm{~cm} / \mathrm{s}$, the mechanical index at 1.5 , the wall filter at $50-100 \mathrm{~Hz}$, and the frame rate at $>50 \mathrm{~Hz}$. Flow gain was increased until noise emerged. When microvessels were detected in SMI, the imaging mode was switched to PDI. SMI that revealed the vasculature by subtracting the background signals for analysis was used.

The method applied by Gokalp et al was used [5] to divide the intraductal breast lesions into subgroups according to their vascularity (fig 1). Based on this grading system, when no flow signals were detected within the lesion, it was classified as avascular; when 1 or 2 vessels were detected within the lesion, it was classified as hypovascular; and when 3 or more vessels were detected within the lesion, it was classified as hypervascular. We assessed the flow distribution mode by location (central

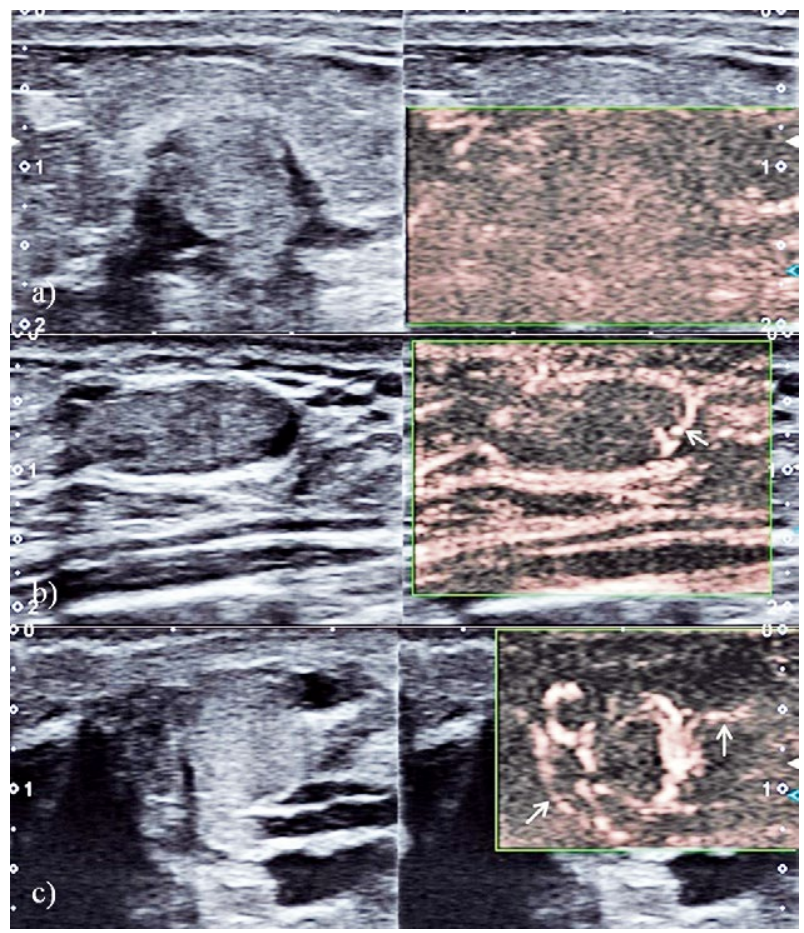

Fig 1. Qualitative evaluation of vascular blood flow in the intraductal breast lesions. Superb microvascular imaging showing no vascularity in the solidified debris (a); hypovascular and peripheral vascular flow in intraductal papilloma (arrow, b); and hypervascular and disordered vascular flow with penetrating vessels in intraductal papillary carcinoma (arrow, c).

and peripheral) and the morphology of flow signals (linear, branching, and disordered). If few regular lines or spots of flow signals appeared within the lesion, we classified it as a linear mode; if flow signals in the lesion were detected with a number of tiny branches, we classified it as a branching mode; and if there were chaotically increased flow signals within the lesion, we classified it as a disordered mode. Penetrating vasculature was defined as flow signals extending from the outside to the inside of the lesion.

\section{Histopathology}

The histopathology of the 54 intraductal breast lesions was confirmed using an US-guided 14-gauge core needle ( $\mathrm{n}=8$, Geotek Medical, Ankara, Turkey) biopsy or surgical excision $(n=46)$. A mean of four core samples were obtained per lesion (range 3-6). Four malignant papillary lesions and 4 atypical lesions diagnosed with core biopsy were treated with surgical excision. We considered the pathology results of surgical excision as the final diagnosis. Histopathologic results were evaluated by a pathologist with 13 years of experience in breast pathology. All intraductal lesions were divided into 3 groups according to the pathologic classification. Group 1 was 
classified as benign intraductal lesions, group 2 was classified as atypical intraductal lesions, group 3 was classified as malignant intraductal lesions.

\section{Statistical analysis}

All statistical analyses were performed with SAS version 9.4 software (SAS Institute Inc, Cary, NC). Categorical variables were compared using the Fisher's exact test. Kruskal-Wallis one-way analysis of variance test was used to compare continuous variables. The McNemar test was used to compare effectiveness of detecting flow signals between SMI and PDI. Area under receiver operating characteristic curve (AUROC) analysis was performed to evaluate the predictive abilities of SMI and PDI. To distinguish benign lesions from malignant and atypical lesions, the sensitivity, specificity, positive predictive value (PPV), negative predictive value (NPV), and accuracy were calculated using cutoff points. The $\mathrm{k}$-coefficient was used to evaluate the inter-rater agreement for SMI and PDI. A p value $<0.05$ was considered statistically significant.

\section{Results}

\section{Patients}

Among the 54 intraductal breast lesions, 39 were benign, 10 were atypical, and 5 were malignant. The histopathologic diagnoses are summarized in Table I. The most frequently encountered clinical presentation was a palpable mass; the second most frequently encountered presentation was nipple discharge that was typically uniorificial and bloody or serous stained. No difference was observed in the location of lesions between the groups.

\section{B-mode ultrasonography}

The mean size of the lesions calculated according to the longest axis was $12.2 \pm 7.2 \mathrm{~mm}$ (range $5-49 \mathrm{~mm}$ ). An oval or round shape and circumscribed margin were the most frequent findings in benign lesions. These shapes and circumscribed margins were found in $9.2 \%$ (5 of 10) of the atypical lesions and $1.8 \%(1$ of 5$)$ of the malignant
Table I. Histopathologic analysis of the intraductal breast lesions

\begin{tabular}{ll}
\hline Benign & \\
Intraductal papilloma & 14 \\
Fibrocystic change & 7 \\
Usual ductal hyperplasia & 3 \\
Fibrosis & 2 \\
$\quad$ Solidified debris & 13 \\
Atypical & \\
$\quad$ Papilloma with atypical ductal hyperplasia & 3 \\
$\quad$ Atypical ductal hyperplasia & 4 \\
Atypical lobular hyperplasia & 3 \\
Malignant & \\
$\quad$ Intraductal papillary carcinoma & 4 \\
İnvasive papillary carcinoma & 1 \\
Total & 54 \\
\hline
\end{tabular}

lesions. Hypoechogenicity was the most common echo pattern in all intraductal lesions. Posterior shadowing was only found in an atypical lesion. Calcification was detected in benign lesions at rate of 5.5\% (3 of 39), in atypical lesions at rate of $3.7 \%$ ( 2 of 10 ), and in malignant lesions at rate of $1.8 \%$ ( 1 of 5 ). While ductal dilatation was detected at a higher rate in malignant and atypical lesions (12 of 15), it was detected at a lower rate in benign lesions (22 of 39).

Most of the benign (30 of 39) and atypical (6 of 10) lesions were classified as BI-RADS category $4 \mathrm{a}$. The other 9 benign lesions, 3 atypical and 2 malignant breast lesions were classified as BI-RADS category $4 \mathrm{~b}$. One atypical and 2 malignant breast lesions were classified as BI-RADS category $4 \mathrm{c}$. The last malignant breast lesion was classified as BI-RADS category 5. The sonographic appearances of the intraductal lesions are summarized in Table II.

\section{Doppler US}

SMI was more sensitive than PDI for detecting subtle blood flow in intraductal breast lesions $(\mathrm{p}=0.004)$. Flow signals were detected in 14 of 39 benign lesions, in 8 of

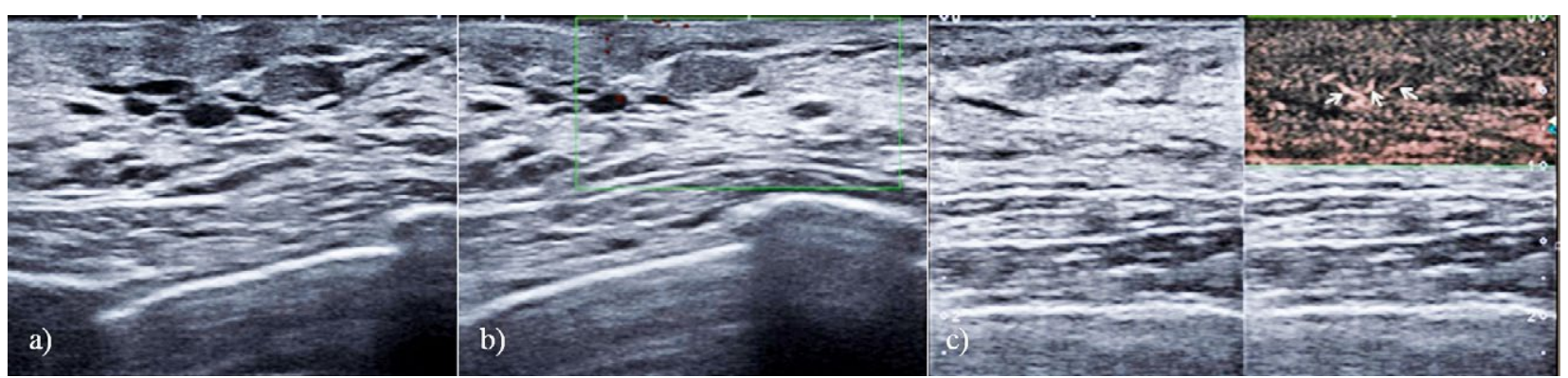

Fig 2. A 47-year-old woman with intraductal papilloma. B-mode ultrasonography showing a well-circumscribed oval mass in the retroareolar region (a). Power Doppler image evidencing no vascularity (b). Superb microvascular imaging showing hypervascularity with branching vessels (arrow, c). 
Med Ultrason 2018; 20(3): 306-312

\begin{tabular}{|c|c|c|c|c|}
\hline & Benign $(n=39)$ & Atypical $(\mathrm{n}=10)$ & Malignant ( $(n=5)$ & $\mathbf{p}$ \\
\hline \multicolumn{5}{|l|}{ Clinical presentation } \\
\hline Palpable mass & $14(25.9)$ & $4(7.4)$ & $3(5.5)$ & \multirow{4}{*}{0.71} \\
\hline Nipple discharge & $10(18.5)$ & $3(5.5)$ & $4(7.4)$ & \\
\hline Breast pain & $8(14.8)$ & $2(3.7)$ & $1(1.8)$ & \\
\hline Screening/Asymptomatic & $11(20.3)$ & $2(3.7)$ & $0(0)$ & \\
\hline \multicolumn{5}{|l|}{ Sonographic Findings } \\
\hline \multicolumn{5}{|l|}{ Shape } \\
\hline Oval, Round & $30(55.5)$ & $5(9.2)$ & $1(1.8)$ & \multirow[t]{2}{*}{0.0196} \\
\hline Irregular & $9(16.6)$ & $5(9.2)$ & $4(7.4)$ & \\
\hline \multicolumn{5}{|l|}{ Margin } \\
\hline Circumscribed & $29(53.7)$ & $5(9.2)$ & $1(1.8)$ & \multirow[t]{2}{*}{0.0347} \\
\hline Not circumscribed & $10(18.5)$ & $5(9.2)$ & $4(7.4)$ & \\
\hline \multicolumn{5}{|l|}{ Echo pattern } \\
\hline Hyperechoic/ isoechoic & $11(20.3)$ & $2(3.7)$ & $2(3.7)$ & \multirow[t]{2}{*}{0.694} \\
\hline Hypoechoic & $28(51.8)$ & $8(14.8)$ & $3(5.5)$ & \\
\hline \multicolumn{5}{|l|}{ Posterior feature } \\
\hline None & $24(44.4)$ & $5(9.2)$ & $4(7.4)$ & \multirow[t]{3}{*}{0.38} \\
\hline Enhancement & $15(27.7)$ & $4(7.4)$ & $1(1.8)$ & \\
\hline Shadowing & $0(0)$ & $1(1.8)$ & $0(0)$ & \\
\hline \multicolumn{5}{|l|}{ Calcifications } \\
\hline Absent & $26(48.1)$ & $8(14.8)$ & $4(7.4)$ & \multirow[t]{2}{*}{0.512} \\
\hline Present & $3(5.5)$ & $2(3.7)$ & $1(1.8)$ & \\
\hline \multicolumn{5}{|l|}{ Ductal dilatation } \\
\hline Absent & $17(31.4)$ & $2(3.7)$ & $1(1.8)$ & \multirow[t]{2}{*}{0.37} \\
\hline Present & $22(40.7)$ & $8(14.8)$ & $4(7.4)$ & \\
\hline \multicolumn{5}{|l|}{ Location } \\
\hline Right & $19(35.1)$ & $6(11.1)$ & $3(5.5)$ & \multirow[t]{2}{*}{0.824} \\
\hline Left & $20(37)$ & $4(7.4)$ & $2(3.7)$ & \\
\hline Size $(\mathrm{mm})$ & $11.5 \pm 4.8$ & $9.7 \pm 5.5$ & $22.8 \pm 15.4$ & 0.015 \\
\hline \multicolumn{5}{|l|}{ SMI blood flow } \\
\hline Absent & $18(33.3)$ & $0(0)$ & $0(0)$ & \multirow[t]{2}{*}{0.003} \\
\hline Present & $21(38.8)$ & $10(18.5)$ & $5(9.2)$ & \\
\hline \multicolumn{5}{|l|}{ PDI blood flow } \\
\hline Absent & $25(46.2)$ & $2(3.7)$ & $0(0)$ & \multirow[t]{2}{*}{0.002} \\
\hline Present & $14(25.9)$ & $8(14.8)$ & $5(9.2)$ & \\
\hline
\end{tabular}

10 atypical lesions, and in all malignant lesions with PDI, whereas they were detected in 21 of 39 benign lesions, and in all atypical and malignant lesions in SMI.

Benign lesions had more frequently avascular (18 of 39 in SMI, 25 of 39 in PDI) and hypovascular patterns (15 of 39 in SMI, 9 of 39 in PDI). These lesions were pathologically diagnosed as intraductal papilloma (fig 2). Most of the atypical lesions were defined as hypervascular with both methods (8 of 10 in SMI, 5 of 10 in PDI). All the malignant lesions were hypervascular in both methods.

While more central vascular distribution was detected in malignant and atypical lesions with both methods, peripheral vascular distribution was more frequent in benign lesions. Further, whereas branching and disordered flow patterns were greater in malignant and atypical lesions, a linear flow pattern was more common in benign lesions..

Penetrating vessels were detected in 4 benign lesions, in 6 atypical lesions, and in all of the malignant legions with the SMI method. However, penetrating vessels were detected in 4 benign lesions, in 4 atypical lesions, and in 4 malignant lesions with the PDI method.

The vascularity features of the lesions and their diagnostic performances (sensitivity, specificity, positive predictive value, negative predictive value, area under receiver operating characteristic curve) are shown in Table III and Table IV. The k-coefficient indicating inter-rater agreement was 0.80 for SMI and 0.76 for PDI. There was a good agreement between the 2 independent operators. 
Table III. Superb microvascular imaging (SMI) and power Doppler imaging (PDI) blood flow characteristics of intraductal breast lesions

\begin{tabular}{|c|c|c|c|c|}
\hline & Benign $(n=39)$ & Atypical $(n=10)$ & Malignant $(n=5)$ & $\mathbf{p}$ \\
\hline \multicolumn{5}{|l|}{ SMI } \\
\hline \multicolumn{5}{|l|}{ Vascular Grading } \\
\hline Avascular & $18(33.3)$ & $0(0)$ & $0(0)$ & \multirow[t]{3}{*}{$<0.0001$} \\
\hline Hypovascular & $15(27.7)$ & $2(3.7)$ & $0(0)$ & \\
\hline Hypervascular & $6(11.1)$ & $8(14.8)$ & $5(9.2)$ & \\
\hline \multicolumn{5}{|c|}{ Vascular distribution } \\
\hline Central & $6(11.1)$ & $7(12.9)$ & $5(9.2)$ & \multirow[t]{2}{*}{0.004} \\
\hline Peripheral & $15(27.7)$ & $3(5.5)$ & $0(0)$ & \\
\hline \multicolumn{5}{|l|}{ Flow pattern } \\
\hline Linear & $14(25.9)$ & $2(3.7)$ & $0(0)$ & \multirow[t]{3}{*}{0.003} \\
\hline Branching & $5(9.2)$ & $5(9.2)$ & $1(1.8)$ & \\
\hline Disordered & $2(3.7)$ & $3(5.5)$ & $4(7.4)$ & \\
\hline \multicolumn{5}{|c|}{ Penetrating vessels } \\
\hline Absent & $22(40.7)$ & $4(7.4)$ & $0(0)$ & \multirow[t]{2}{*}{0.0001} \\
\hline Present & $4(7.4)$ & $6(11.1)$ & $5(9.2)$ & \\
\hline \multicolumn{5}{|l|}{$P D I$} \\
\hline \multicolumn{5}{|l|}{ Vascular grading } \\
\hline Avascular & $25(46.2)$ & $2(3.7)$ & $0(0)$ & \multirow[t]{3}{*}{0.0001} \\
\hline Hypovascular & $9(16.6)$ & $3(5.5)$ & $0(0)$ & \\
\hline Hypervascular & $5(9.2)$ & $5(9.2)$ & $5(9.2)$ & \\
\hline \multicolumn{5}{|c|}{ Vascular distribution } \\
\hline Central & $4(7.4)$ & $4(7.4)$ & $5(9.2)$ & \multirow[t]{2}{*}{0.017} \\
\hline Peripheral & $10(18.5)$ & $4(7.4)$ & $0(0)$ & \\
\hline \multicolumn{5}{|l|}{ Flow pattern } \\
\hline Linear & $9(16.6)$ & $3(5.5)$ & $0(0)$ & \multirow[t]{3}{*}{0.050} \\
\hline Branching & $3(5.5)$ & $3(5.5)$ & $1(1.8)$ & \\
\hline Disordered & $2(3.7)$ & $2(3.7)$ & $4(7.4)$ & \\
\hline \multicolumn{5}{|l|}{ Penetrating vessels } \\
\hline Absent & $24(44.4)$ & $6(11.1)$ & $1(1.8)$ & \multirow[t]{2}{*}{0.001} \\
\hline Present & $2(3.7)$ & $4(7.4)$ & $4(7.4)$ & \\
\hline
\end{tabular}

The results are presented as number (\%).SMI, superb microvascular imaging; PDI, power Doppler imaging

Table IV. Diagnostic effectiveness of different vascular grading methods in power Doppler imaging (PDI) and superb microvascular imaging (SMI)

\begin{tabular}{|c|c|c|c|c|c|}
\hline & AUROC & SEN (\%) & SPE (\%) & PPV (\%) & NPV (\%) \\
\hline \multicolumn{6}{|l|}{ SMI } \\
\hline Vascular grading & 0.862 & 86.6 & 84.6 & 68.4 & 94.2 \\
\hline Flow distribution & 0.804 & 80 & 80.7 & 70.5 & 87.5 \\
\hline Penetrating vessels & 0.790 & 73.3 & 84.6 & 73.3 & 84.6 \\
\hline \multicolumn{6}{|l|}{ PDI } \\
\hline Vascular grading & 0.773 & 66.6 & 87.1 & 66.6 & 87.1 \\
\hline Flow distribution & 0.742 & 60 & 88.4 & 75 & 79.3 \\
\hline Penetrating vessels & 0.728 & 53.3 & 92.3 & 80 & 77.4 \\
\hline
\end{tabular}

AUROC, area under receiver operating characteristic curve; SEN, sensitivity; SPE, specificity; PPV, positive predictive value; NPV, negative predictive value; SMI, superb microvascular imaging; PDI, power Doppler imaging

\section{Discussions}

Intraductal breast lesions are an unusual group of breast diseases that display unique diagnostic and management challenges because of a wide spectrum of imag- ing appearances [8]. In the evaluation of intraductal breast lesions, mammography and galactography are gradually being replaced by US and magnetic resonance imaging (MRI) examinations with their significantly higher resolutions $[9,10]$. High-frequency US is the most widely 
preferred imaging method in the evaluation of breast lesions. Because of its high resolution, shape, margin, and echo pattern, calcifications and adjacent tissue changes can be easily evaluated [11]. However, there is a wide spectrum of appearances of intraductal breast lesions on different imaging modalities. This variable appearance of intraductal lesions renders the differentiation of benign from malignant pathologies difficult on imaging $[8,11]$. For this reason, the sensitivity of both US and MRI methods in the differentiation of intraductal breast lesions is high but their specificity is low $[8,12,13]$. The diagnosis of lesions is usually made using tissue sampling.

Tumor angiogenesis is useful for discriminating benign from malignant tumors. The detection of vascularization in the lesions is significantly associated with malignancy [14,15]. However, conventional Doppler methods are limited by their angular dependency and poor signal-to-noise ratios, and they often fail to evaluate flow signals from small vessels [16]. Recently, a new Doppler ultrasound technique, called SMI, was developed to overcome these limitations for microvessel evaluation. SMI exposed more details of the microvasculature, such as tiny branches and the distal part of microvessels. SMI can separate low-speed flow signals from motion artifacts [13]. Thus, more flow signals were obtained from the lesions. In a study, Ma et al detected more blood flow and flow detail than color Doppler imaging in evaluation malignant and benign breast lesions [17]. Similarly in our study, we detected vascularity in more intraductal lesions and obtained more flow distribution detail with the SMI method.

Previous studies reported that PDI was useful in distinguishing benign breast lesions from malignant breast lesions [18-20]. It was shown that malignant breast lesions are more hypervascular, while benign breast lesions are more avascular or hypovascular. It was also reported that lesions with central distribution, more branching, and irregular vessels were more likely to be malignant $[3,21]$. Yongfeng et al detected hypervascularization in 33 of 41 malignant lesions using the SMI method (80.4\%). Further, more central distribution patterns and more branching and disordered vessels were detected in malignant lesions in their study [13]. While we detected hypervascularization in $86.6 \%$ of all atypical and malignant intraductal lesions with SMI method in our study, this rate was $66.6 \%$ with the PDI method. Moreover, the central flow pattern in atypical and malign intraductal lesions was $80 \%$ with the SMI method in our study, whereas this rate was $60 \%$ with the PDI method.

The presence of penetrating vessels is another important feature in the evaluation of malignant lesions. Previous studies of PDI supported the fact that penetrating vessels play an important role in the diagnosis of breast lesions. Their sensitivity for the diagnosis of breast malignancy ranged from $56 \%$ to $68 \%$ and their specificity ranged from $72 \%$ to $95 \%[22,23]$. In our study, we detected more penetrating vessels in atypical and malignant intraductal lesions with the SMI method. Using penetrating vessels, we achieved $73.3 \%$ sensitivity and $84.6 \%$ specificity with the SMI method for the differentiation of atypical and malignant lesions from benign intraductal breast lesions, while we obtained $53.3 \%$ sensitivity and 93.3\% specificity with PDI.

Another important goal of our study was to distinguish solidified intraductal debris, which is composed of intraluminal acidophilic material and foamy macrophages, from real solid lesions. These benign lesions are seen more widely and it cannot always be easily distinguished with B-mode US. When debris is mobile or in fluid form and does not show blood flow with any Doppler US method, it can be easily identified [24]. However, when it is solidified and immobile, it can mimic intraductal papilloma [25]. In such a case, it is difficult to determine whether it is an avascular or hypovascular solid lesion or simple intraductal debris. For this reason, unnecessary biopsies or advanced radiological imaging methods, such as MRI, are applied for these lesions. In our study, we achieved more sensitivity with SMI than PDI for detecting subtle blood flow in intraductal breast lesions. So, SMI has better diagnostic accuracy for distinguishing real solid lesions from solidified debris.

This study has some limitations. The sample size was relatively small, and the variation of histopathologic types was also limited. Additional studies with larger populations are required.

\section{Conclusion}

SMI is more sensitive than PDI in detecting microvascular blood flow and vascular distribution in intraductal breast lesions with statistical significance. This promising vascular imaging technique can help B-mode US to distinguish intraductal breast lesions more accurately.

\section{Conflict of interest: none}

\section{References}

1. Ganesan S, Karthik G, Joshi M, Damodaran V. Ultrasound spectrum in intraductal papillary neoplasms of breast. Br J Radiol 2006;79:843-849.

2. Shin HJ, Kim HH, Kim SM, et al. Papillary lesions of the breast diagnosed at percutaneous sonographically guided biopsy: comparison of sonographic features and biopsy methods. AJR Am J Roentgenol 2008;190:630-636. 
3. Stanzani D, Chala LF, Barros Nd, Cerri GG, Chammas MC. Can Doppler or contrast-enhanced ultrasound analysis add diagnostically important information about the nature of breast lesions? Clinics (Sao Paulo) 2014;69:87-92.

4. Fox SB, Generali DG, Harris AL. Breast tumour angiogenesis. Breast Cancer Res 2007;9:216.

5. Gokalp G, Topal U, Kizilkaya E. Power Doppler sonography: anything to add to BI-RADS US in solid breast masses? Eur J Radiol 2009;70:77-85.

6. Arslan S, Karahan AY, Oncu F, Bakdik S, Durmaz MS, Tolu I. Diagnostic Performance of Superb Microvascular Imaging and Other Sonographic Modalities in the Assessment of Lateral Epicondylosis. J Ultrasound Med 2018;37:585-593.

7. Artul S, Nseir W, Armaly Z, Soudack M. Superb Microvascular Imaging: Added Value and Novel Applications. J Clin Imaging Sci 2017;7:45.

8. Brookes MJ, Bourke AG. Radiological appearances of papillary breast lesions. Clin Radiol 2008;63:1265-1273.

9. Le-Petross CH, Bidaut L, Yang WT. Evolving role of imaging modalities in inflammatory breast cancer. Semin Oncol 2008;35:51-63.

10. Ferris-James DM, Iuanow E, Mehta TS, Shaheen RM, Slanetz PJ. Imaging approaches to diagnosis and management of common ductal abnormalities. Radiographics 2012;32:1009-1030.

11. Eiada R, Chong J, Kulkarni S, Goldberg F, Muradali D. Papillary lesions of the breast: MRI, ultrasound, and mammographic appearances. AJR Am J Roentgenol 2012;198:264271.

12. Choi JJ, Kang BJ, Kim SH, et al. Role of sonographic elastography in the differential diagnosis of papillary lesions in breast. Jpn J Radiol 2012;30:422-429.

13. Yongfeng Z, Ping Z, Wengang L, Yang S, Shuangming T. Application of a Novel Microvascular Imaging Technique in Breast Lesion Evaluation. Ultrasound Med Biol 2016;42:2097-2105.

14. del Cura JL, Elizagaray E, Zabala R, Legorburu A, Grande D. The use of unenhanced Doppler sonography in the evaluation of solid breast lesions. AJR Am J Roentgenol 2005;184:1788-1794.

15. Zhu Q, Cronin EB, Currier AA, et al. Benign versus malignant breast masses: optical differentiation with US-guided optical imaging reconstruction. Radiology 2005;237:57-66.

16. Schroeder RJ, Bostanjoglo M, Rademaker J, Maeurer J, Felix R. Role of power Doppler techniques and ultrasound contrast enhancement in the differential diagnosis of focal breast lesions. Eur Radiol 2003;13:68-79.

17. Ma Y, Li G, Li J, Ren WD. The Diagnostic Value of Superb Microvascular Imaging (SMI) in Detecting Blood Flow Signals of Breast Lesions: A Preliminary Study Comparing SMI to Color Doppler Flow Imaging. Medicine (Baltimore) 2015;94:e1502.

18. Cosgrove DO, Kedar RP, Bamber JC, et al. Breast diseases: color Doppler US in differential diagnosis. Radiology 1993;189:99-104.

19. Adler DD, Carson PL, Rubin JM, Quinn-Reid D. Doppler ultrasound color flow imaging in the study of breast cancer: preliminary findings. Ultrasound Med Biol 1990;16:553-559.

20. Kook SH, Park HW, Lee YR, Lee YU, Pae WK, Park YL. Evaluation of solid breast lesions with power Doppler sonography. J Clin Ultrasound 1999;27:231-237.

21. Milz P, Lienemann A, Kessler M, Reiser M. Evaluation of breast lesions by power Doppler sonography. Eur Radiol 2001;11:547-554.

22. Raza S, Baum JK. Solid breast lesions: evaluation with power Doppler US. Radiology 1997;203:164-168.

23. Svensson WE, Pandian AJ, Hashimoto H. The use of breast ultrasound color Doppler vascular pattern morphology improves diagnostic sensitivity with minimal change in specificity. Ultraschall Med 2010;31:466-474.

24. Hamed ST, Abdo MH, Ahmed HH. Breast discharge: ultrasound and Doppler evaluation. J Egypt Natl Canc Inst 2008;20:262-270

25. Tourasse C, Coulon A, Denier JF. Radio-histological correlations of subtle sonography images. Diagn Interv Imaging 2014;95:181-195. 